\title{
EFFECT OF OCCLUSAL TRAUMA ON HEALING OF PERIAPICAL LESION: (Case Report)
}

\author{
Trimurni Abidin \\ Department of Conservative Dentistry Faculty of Dentistry University of Sumatera Utara
}

\begin{abstract}
Traumatic injury of the teeth occurs frequently on anterior and posterior teeth. Pulp necrosis is often the frequent sequel of trauma and it will lead to development of periapical lesions in the presence of pathogenic microorganism. Two cases of periapical lesion reported here were caused by occlusal trauma. It is still controversial whether a case of large periapical lesion should be treated conventionally or surgically. This cases was treated non surgically and it was proved that this kind of treatment could promote large apical healing successfully. Indonesian Journal of Dentistry 2006; Edisi Khusus KPPIKG XIV: 266-271
\end{abstract}

Key words: occlusal trauma, periapical lesion, non surgical endodontic.

\section{Introduction}

Occlusal trauma is defined as a pathological condition or an adaptive change that involves periodontal tissue as the result of the strength produced by masticatory muscles. ${ }^{1}$ Occlusal trauma can develop into a condition which load strength produced by the occlusion exceeds the ability of periodontal tissue around the teeth that gets involved, also in nonfunctional condition and produces a strength without movement. This exceeding occlusal strength can cause dystrophic in periodontal ligament, aiveolar bone, cementum and also could induces periapical inflammation and root resorption. ${ }^{2}$

The relationship between occlusal trauma and periapical healing has been reported a lot and well established. When the periodontal tissue is heaithy, occlusal trauma may not cause the formation of dental pocket or the destruction of connective tissue attachment. Bacterial infection combined with occlusal trauma can cause damage to the periodontal tissue. In this case, occlusal trauma plays its role as co-factor in destruction process. ${ }^{1}$

Eldeeb and Andreason (1991) has reported their research, using rats as trial animals, that hyper and hypo occlusion did not affect periapical healing. ${ }^{3}$ Kumazawa, et al (1995) reported his research which also used rats as trial animal, the correlation was found between occlusal trauma and periapical pathosis. It was presumed that occlusal trauma could be associated with slow-developing inflammation in periapical tissue ${ }^{4}$.

These two case-reports showed the root canal treatments performed in maxillary premolar teeth and maxillary central incisor that experienced occlusal trauma caused by dental disorder. In one case, the healing took place after occlusal adjustment while in other case healing was not seen. Persistent pain occured eventhough the root canal 
treatment had been well performed. An occlusal adjustment on the teeth could not be performed yet.

\section{Case Report}

Case I

$\Lambda$ female patient, 17 years of age visited dental cliric (Conservative Department in Faculty of Dentistry, University of Sumatera Utara) with the need to have the decayed right maxillary premolar restored because she felt discomfort while eating and sometimes it was throbbing. She also wanted to change her outlook because the dental order was not good. Before this, the tooth had been painful for about past six months but the pain had gone if she took analgesic. When she visited the dental clinic, the tooth was not painful anymore.

In a clinical examination, it was seen that tooth 14 with fracture palatal cusp, and caries with pulp expossure. The remaining tooth structures left were buccal and a few in mesial and distal part. Vitality test of the teeth did not show any sensitivity but it was sensitive to percussion and palpation. Radiographic view showed that the root had shortened, and apical root canal narrowed. The periodontal ligament widened, lamina dura disappeared and wide radioluscency viewed at periapex. (Fig 1). Tooth 14 was found palatoversion in the dental arch (Fig 2). The diagnosis of tooth 14 was pulp necrotic with chronical apical periodontitis. The treatment performed was a non-vital root canal treatment with post core and porcelain full crown to restore the dental occlusion into the dental arch.

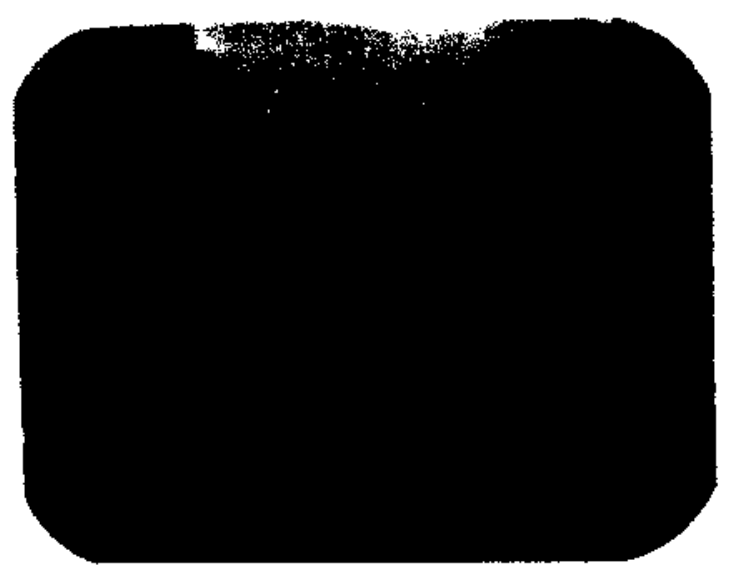

Fig 1. Tooth 14 with shortened root radiographically an apical narrow of its pulp chamber and periapical radiolucency. (2-08-2005).

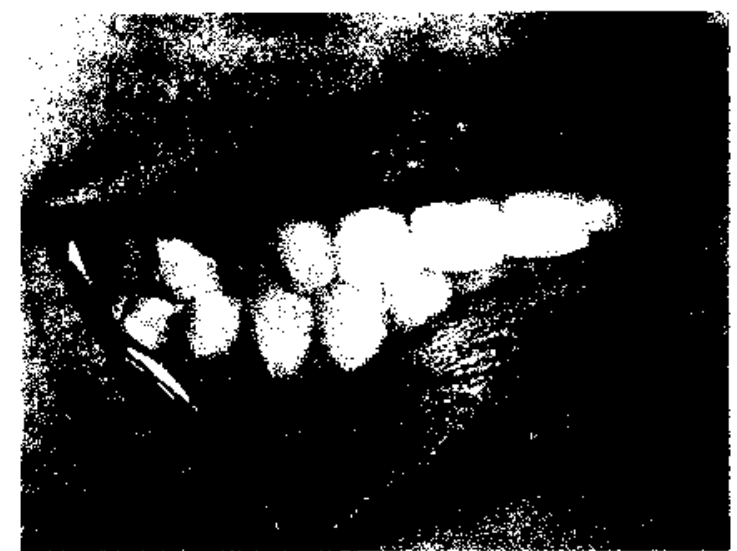

Fig 2. The position of the teeth was palatoversion

Non-vital root canal treatment was performed on tooth 14. After biomechanical root canal preparation, calcium hydroxide as dressing with glycerin as its vehicle was applied to the tooth. The change of intra-canal dressing was performed every week by observing the subjective and objective complaints of the patient. In the root canal preparation, apical reach was hard to attain, though the chelating agent and irrigation with $\mathrm{NaOCl} 2.5 \%$ had been applied. Buccal root was prepared until size/length $35 / 15$ and palatal root was $40 / 13$.

Root canal filling was performed after the third week with gutta-percha by using lateral condensation technique and Apexit (Ivoclar Vivadent, Liechstenstein) as the sealer. In control a week after root canal filling, fabricated post was cemented in buccal root and full porcelain crown was prepared. The cementation of full crown on tooth 14 was completed after two weeks' core cementation by using luting type of Glass Ionomer Cement (Fig 3).

Control was carried out after the sixth month, it was seen that the patient had no complaints, radiographic view showed the diminution of periapical lesion.

\section{Case 2}

A female patient, 36 years of age was referred with pain and swelling of maxillary anterior teeth. The swelling had occurred for a week and this condition had occurred several times. Teeth had ever received root canal treatment.

Clinical examination showed teeth 11 and 21 with Composite Resin filling on the palatal side. Teeth showed grayish-brown discoloration. Palpation on region 11 and 21 showed hypersensitivity to 
percussion, tooth 11 was more sensitive than tooth 21. Radiographic view showed widening of radioluscency in the apical of tooth 11 with nonhermetic root canal filling on both teeth (Fig 5). The occlusal relation of maxillary and mandibular anterior teeth was not good (Fig 6).

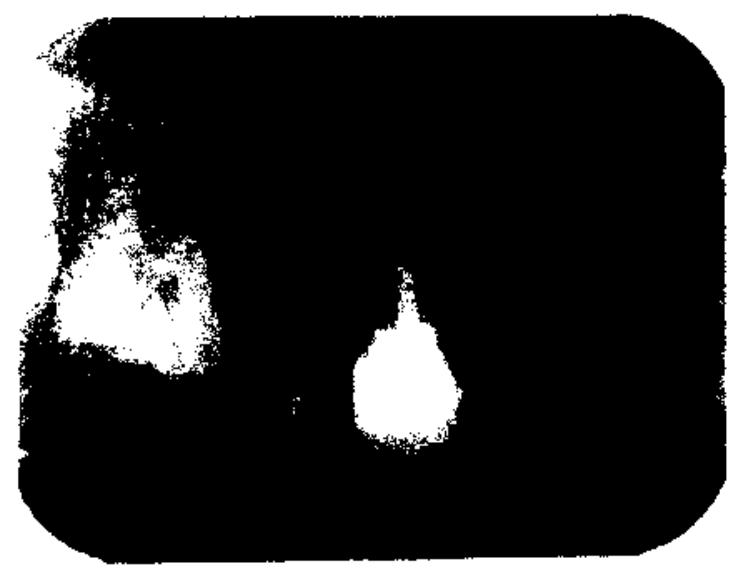

Fig 3. Tooth 14 with post core and full porcelain crown $(9 / 09 / 2005)$

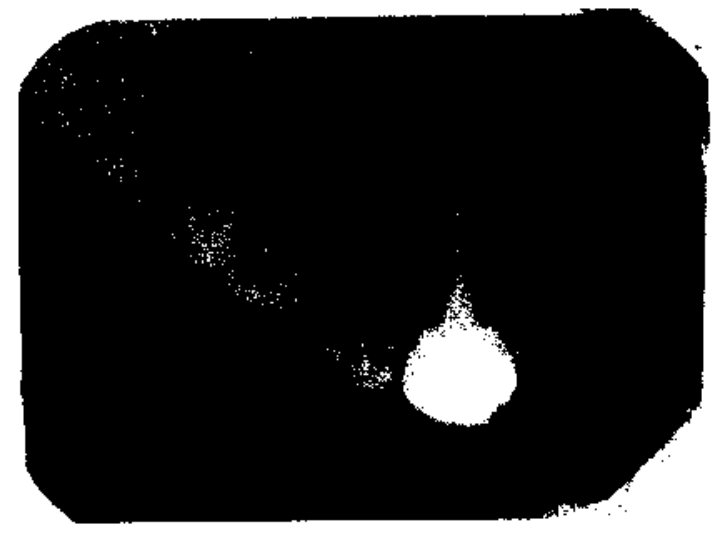

Fig 4 Control on tooth 14 after six month of the treatment. It was seen that there was a diminution of periapical lesion $(28 / 03 / 2006$

Non-surgical endodontic retreatment was performed on both teeth. Biomechanical preparation and irrigation with $\mathrm{NaOCl} 2.5 \%$ were performed on teeth 11 and 21 . Calcium hydroxide was applied after drainage had been completed. Oral pre-medications such as analgesic, anti-inflammation, antibiotic and vitamin were given to the patient.

Four days after the first visit, patient returned with pain on tooth 11 . Treatment was continued with biomechanical preparation, irrigation and calcium hydroxide as dressing. In this second visit, maxillary

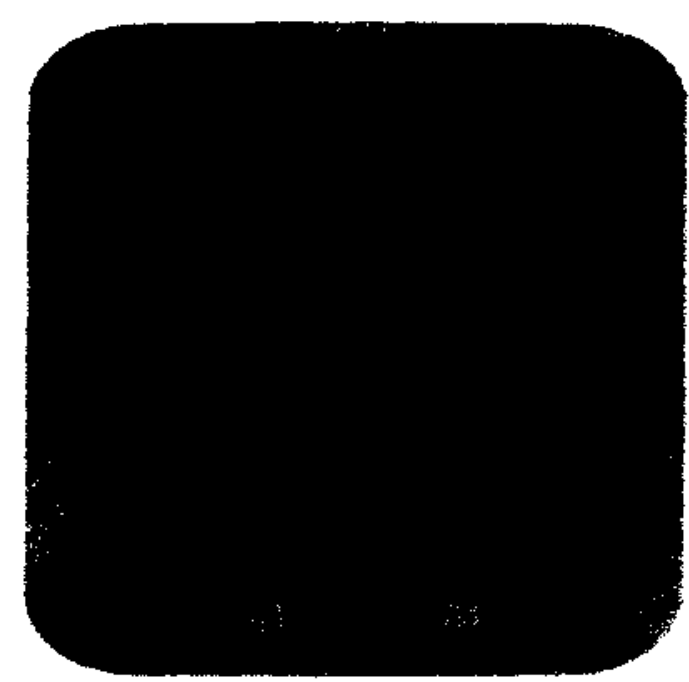

Fig 5. Tecth 11 and 21 with non-hermetic root canal filing and radiolucent view in apex of tooth 11, (21-102005)

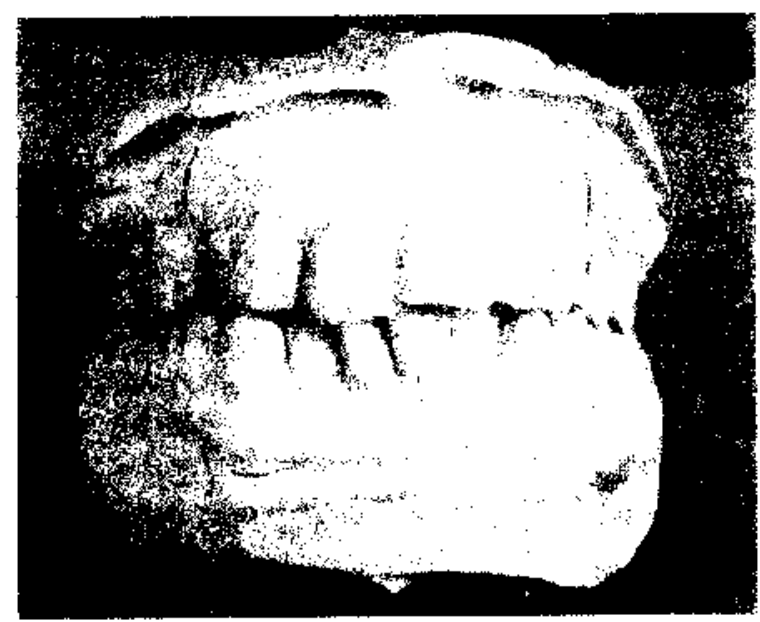

Fig 6 . The relation between teeth 1,21 and mandibular teeth was not good.

and mandibular impressions were taken to observe the patient's occlusal relation. Occlusal adjustment was made on teeth 11 and 21 with antagonist teeth by reducing the occlusal trauma. After this second visit, two days later patient reported that it was better than the first visit. Ten days later, patient was back with swollen on maxillary anterior teeth especially on tooth 11. In this third visit, root canal was opened up and drainage on tooth 11 was completed through its root canal. Biomechanical preparation was performed on tooth 21 and dressing with calcium hydroxide was applied. Tooth was then restored with temporary filling. Pre-medications such as 
performed to observe the patient's occlusal relation. Occlusal adjustment was made on teeth 11 and 21 with antagonist teeth by reducing the occlusal trauma. After this second visit, two days later patient reported that it was better than the first visit. Ten days later, patient was back with swelling on maxiliary anterior teeth especially on tooth 11 . In this third visit, root canal was opened up and drainage on tooth 11 was completed through its root canal. Biomechanical preparation was performed on tooth 21 and dressing with calcium hydroxide was applied. Tooth was then restored with temporary filling. Pre-medications such as Analgesic, antiinflammation and antibiotic were given again with patient's approval. Surgical endodontic treatment (apical curettage) was planned to be performed provided the swelling disappear.

In this fourth visit i.e. a week after the third visit, surgical endodontic treatment was performed. Trapezoidal flap opening and apical curettage were performed in the periapex of tooth 11 (Fig 7). After the periapical cleaning of tooth 11 , flap was sutured to its position.

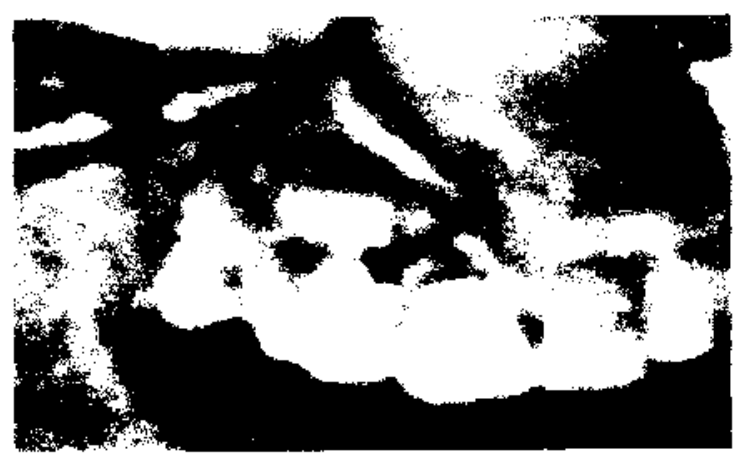

Fig 7. Surgical endodontic treatment on tooth 11 (apical curettage). (15-1 l-2005)

In control that was in the fifth visit, patient reported that she felt discomfort on tooth 11 especially against percussion. Clinicat examination showed good healing on surgical area. Filing through the root canal showed pus and granulation tissues. After the suture was opened, it was decided that the root canal of tooth 11 was left open. The examination on tooth 21 showed no symptoms.

The sixth visit i.e. two weeks after surgery, patient returned with permanent discomfort feeling on tooth 11. Tooth 21 showed no symptoms therefore root canal filling of tooth 21 was performed with sealer containing calcium hydroxide and gutta. percha using condensation technique. The root canal of tooth 11 was still open because pus was still found.

Five days later, control was held again on tooth 11 and apical inflammation disappeared. Filing of the tooth showed no pus but because patient had ever shown the discomfort feeling, root canal was left open. Three weeks after surgery, root canal treatment was performed on tooth 11 . Dressing with calcium hydroxide was applied and tooth was restored with temporary filling.

A month later, patient returned with complaint of discomfort feeling, though no sensitivity to palpation but a little sensitivity to percussion. Endodontic treatment was continued by applying calcium hydroxide and with radiographic control. There was a repair seen in periapex of tooth 11 (Fig 8).

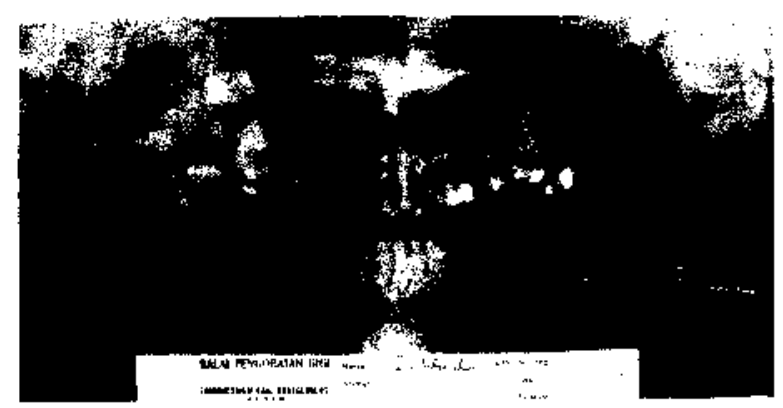

Fig 8. Radiograph of post surgical endodonic trealment of pcriapical tooth 11., (7-2-2006)

Treatment was continued ten days later as well as root canal filling with sealer containing calcium hydroxide and gutta-percha by using lateral condensation technique because patient had no complaints shown as the former visits had.

A month after tilling, radiographic view was seen much better but patient still felt discomfort, especially if the tooth was percussed. In the visit after two months, the examination of tooth 11 showed sensitivity with percussion but tooth 21 had no symptoms. Observation was still carried out on this tooth 11 .

\section{Discussion}

Occlual trauma can occur due to parafunctional habits such as bruxism and clenching or due to incorrect direction of occlusal force when both antagonist of occlusal surfaces encounter. Occlusal trauma can cause the exceeding of occlusal use, fracture of maxillary teeth crown, widening of 
and anaphylactic response (Ig E) such as cytokines produced by lymphocytes, macrophages, connective tissue cells, arachidonic acid metabolites and also kinir produced by other type of cells ${ }^{5}$. The possibility of immune cells infiltration that releases their products may cause periapical bone resorption both in the first and second cases. Chronic inflammation as the result of the occlusal trauma increases bone resorption and bone loss 6,7 .

In the first case, occlusal trauma occurred because the dental position was not in the dental arch (palatoversion). As the result of prolonged occlusal trauma, it was seen that the root shortened with wide periapical lesion. This shortened root showed the external resorption of tooth 14 . The treatment that was performed in this first case was seen to be able to repair the condition of periapex especially by adjusting the dental position with post and crown after non-surgical endodontic treatment was completed.

In the second case, it was seen that there was an exceeding contact relation on teeth 11 and 21 against their antagonist teeth so that periapical lesion might occur. Initial treatment that had been performed by the former clinician showed non-hermetic root canal filling and when the patient came in the first visit there was abscess seen on region 11 and 21 . Nonsurgical retreatment showed that in every cavity sealing on tooth 11 patient had swelling and pain again especially on tooth 11 . Compared to tooth 21 , this tooth showed no reactions and treatment could be performed easily until the root canal filling.

Looking at the condition of tooth 11 , it was decided to perform surgical endodontic treatment to eliminate the periapical lesion and relieve the patient's pain. In the flap opening, it was seen that the lesion widened from mesial of apical tooth 11 until mesial of tooth 12. Cavity was left open for several days after the surgical endodontic treatment until the next examination and was not found any pus or blood in the root canals. The treatment was continued until the mesial of the root canals and the swelling had gone. A few months after the root canal filling, patient remained discomfort and percussion on tooth 11 was more sensitive than on teeth 21 and 12 .

In radiographic control, it was seen that there was a repair in periapical lesion of tooth 11 and radiolucent view showed smaller in six months. The discomfor feit by the patient was presumed caused by maxillary and mandibular anterior teeth that had incorrect relation so that the mechanical irritation could remain. The continuous trauma could retard healing even though radiographic view showed the diminution of the lesion, but the process that occurred in the apical area could not be explained.

Spasser and Wendt (1973 cit Boucher, et al 2000) explained this persistent pain phenomenon could be associated with bone defect. This case might occur due to the prolonged mechanical irritation besides the non-hermetic filling in the root canal treatment that had been performed. Both bacterial and mechanical irritation process could occur on tooth $11^{8}$. Yamaguchi and Kasai (2005) also explained that the incident of pain could be associated with the interaction of inflammatory mediators. Hence the control of inflammatory mediators constituted the main role in pain relief ${ }^{9}$.

Though the irritation reduction as the result of the trauma had been performed, the repair of patient's dental order in the second case constituted the most essential thing to the healing in this case. It was expected that the good root canal treatment and hermetic root canal filling as well as the elimination of mechanical irritation enabled the healing of periapical lesion on tooth 11 and so relieved the pain or patient's discomfort.

\section{Conclusion}

Occlusal trauma can cause inflammatory reaction in periapical tissue that may cause periapical lesion. Non-surgical endodontic treatment can be perfonned in cases of inflamed pulp and periapex as the result of occlusal trauma and it is seen that there was healing as long as the trauma can be eliminated.

The findings in these two cases suggest that in some circumstances, occlusal trauma may play a role in the healing of periapical pathosis.

\section{References}

1. Lindhe J, Nyman $\mathrm{S}$, and Ericsson 1. Trauma From Occlusion In: Lindhe J. Karreng T. Lang N.P. (eds), Clinical Periodontology and Implant Dentistry. $3^{\text {rd }} \mathrm{ed}$. Copenhagen : Munksgaard ,1998: 279-94.

2. Neff P. Trauma From Occlusion; Restorative Concerns. Dent Clin North Am 1995; $39: 335$ - 54

3. Eldeeb $\mathrm{ME}$, Andreasen JO. Histometric study of the Effect of Occiusal Alteration on Periodontal Tissue Healing after Surgical Injury. Endodontics and Dental Traumatology, $1991: 7: 158-63$.

4. Kumazawa M, Kohsaka T, Yamasahi M, Nakamura $\mathrm{H}$, Kameyama Y. Effect of Traumatic Occlusion in Periapical Lesion in Rats. $J$ Endo 1995 ; 21:372- 5. 
5. Stashenko P, Teles R, D'Souza R. Periapical Inflammatory Responses and their Modulation. Crit Rev Oral Biol Med. $1998 ; 9: 498-52$.

6. Barkhodar RA, Zamir Hussain $M$, Hayashi $C$. Detection of Interleukin - 1 Beta in Human Periapical Lesions, Oral Surg Oral Med Oral Pathol 1992;73: 334-6.

7. Nair PNR. Pathobiology of the Periapex. In: Cohen S. and Burns R(eds): Pathway of the Pulp $8^{\text {th }}$ eds. St Louis: Mosby, 2002: 457-500.
8. Boucher Y, Sobel M, Sauveur G. Persistent Pain Related to Root Canal Filling and Apical Fenestration: A Case Report. J Endodon 2001; 28 : 242-4.

9. Yamaguchi M, Kasai K. Inflammation in Periodontal Tissues in Response to Mechanical Forces. Arch Immunol Ther Exp 2005; 53:388-98. 